\title{
Mobile Devices and Insomnia: Understanding Risks and Benefits
}

\author{
Mohammed N. Khan $^{1} \cdot$ Rebecca Nock ${ }^{2} \cdot$ Nalaka S. Gooneratne ${ }^{3}$
}

Published online: 19 October 2015

(C) Springer International Publishing AG 2015

\begin{abstract}
Mobile devices (smartphones and tablet computers) have become widely prevalent due to rapid improvements in function and decreasing costs. As of $2014,90 \%$ of US adults have a mobile phone, with $58 \%$ having a smartphone, $32 \%$ owning some type of e-reader, and $42 \%$ of US adults owning a tablet computer. Mobile devices are particularly well-suited for the study of common conditions such as sleep difficulties because of their ubiquity. Around 35 to $49 \%$ of the US adult population have problems falling asleep or have daytime sleepiness. These sleep disorders are often under-recognized because of patient-physician communication difficulties, low rates of medical awareness resulting in underreporting of insomnia symptoms, and limited primary care physician (PCP) training in insomnia recognition. Mobile devices have the potential to bridge some of these gaps, but they can also lead to sleep difficulties when used inappropriately.
\end{abstract}

Keywords Insomnia recognition - Insomnia treatment . Mobile device therapy · Sleep apps · Insomnia epidemiology .

This article is part of the Topical Collection on Sleep Epidemiology

Mohammed N. Khan

tuf31018@temple.edu

Rebecca Nock

rnock@nursing.upenn.edu

Nalaka S. Gooneratne

ngoonera@mail.med.upenn.edu

1 College of Public Health, Temple University, Philadelphia, PA, USA

2 School of Nursing, University of Pennsylvania, Philadelphia, PA, USA

3 Division of Geriatric Medicine and Center for Sleep and Circadian Neurobiology, University of Pennsylvania, Philadelphia, PA, USA
Sleep diaries $\cdot$ Circadian timing $\cdot$ Snore detection $\cdot$ Relaxation apps · Mobile device use - Mobile device determinants . Insomnia correlates $\cdot$ Mobile device correlates and insomnia prevention

\section{Introduction}

Over the past decade, the declining cost and increasing computational power of mobile devices, such as smartphones and tablet computers, has created a revolution in personal communications [1]. In 2013, it is estimated that there were 181.4 million smartphone users alone, up from 115.1 million in 2012 [2]. Not surprisingly, mobile devices have become increasingly commonplace in healthcare settings. One study of emergency room patients noted that $82 \%$ had cell phones, with $90 \%$ of the cell phone owners having the device with them during their emergency room visit [3•]. An increasing number of clinical research studies have also been conducted with mobile devices.

There are several factors underlying the potential utility of mobile devices in healthcare. First, they are ubiquitous in an individual person's life: the average smartphone is used for 132 min per day, with the majority of that time spent accessing the internet or e-mail. Indeed, only $16 \%$ of that time is spent making phone calls [2]. Second, mobile devices have a remarkable ease of access due to instant-on technology: $80 \%$ of smartphone users will turn the smartphone on within 15 min of waking up [2]. Third, mobile devices have the ability to deliver personalized contact through internet access and the expanding computational power of the device. Fourth, the decreasing cost of mobile device ownership have made them accessible to individuals from a diverse range of socioeconomic backgrounds: even amongst those with annual income less than $\$ 20,000$, cell phone ownership rates are as high 
as $80 \%$ suggesting that socioeconomic status is not an absolute barrier to mobile device access [3•].

As of January 2014, the Pew Research Center noted that $90 \%$ of American adults have a mobile phone, $58 \%$ of American adults have a smartphone, $32 \%$ of American adults own some type of e-reader, and $42 \%$ of American adults own a tablet computer. Based on gender, $92 \%$ of men and $88 \%$ of women have a mobile phone. Ethnically, $92 \%$ of Hispanics, $90 \%$ of African-Americans, and $90 \%$ of whites own a mobile phone [4].

Of the $58 \%$ of all adults who own a smartphone, there is an approximately equal prevalence between men and women. There are, however, significant age differences in rates of ownership: $19 \%$ of Americans aged 65 and older own smartphone whereas $49 \%$ of Americans ages 50 to 64 years own one. In the age group between 30 and 49 years, $74 \%$ own a smartphone. The highest percentage of smartphone owners is seen in the age group between 18 and 29 years with $83 \%$ [4].

In terms of Socioeconomic Status (SES), $44 \%$ of the households earning less than $\$ 30,000$ per year own a smartphone whereas $53 \%$ own a smartphone when income rises to $\$ 49,999$ per year. When income is between $\$ 50,000$ and $74,999,61 \%$ of the households have access to a smartphone. Once the annual household income rises above $\$ 75,000,81 \%$ of the households own a smartphone device [4]. However, in order to fully understand the use of mobile devices, additional categories of mobile devices need to be examined. Devices such as watches, smart fabrics, sensors, and other wearables need to be studied in future research.

The broad ownership of mobile devices across age, race, gender, and socioeconomic status make these devices particularly well-suited for the study of common conditions, such as insomnia or other sleep disorders. Qualitative research examining patient preferences for insomnia assessment and treatment has shown a keen interest in and acceptance of the use of mobile devices $[5 \bullet]$.

\section{Insomnia Epidemiology and Recognition}

Insomnia disorder is defined in the Diagnostic and Statistical Manual-V (DSM-V) [6] as characterized by difficulty falling asleep, staying asleep, or early morning awakenings despite the opportunity to get adequate sleep, leading to significant distress. Insomnia can result in low energy levels, mood swings, poor quality of life and work performance, and feeling unrefreshed upon awakening [7]. The three main factors regulating sleep are as follows: homeostatic factor (average level of sleep depth, duration of prior sleep, and wakefulness), endogenous circadian factor (24-h cycle interacts with environmental light-dark cycle), and a behavioral factor [8•]. Mobile devices use may influence behavioral factors primarily, but can also act on the other two domains relative to sleep. Behavioral factors, in particular, have the potential to override the other two factors if certain behaviors carried out close to sleep can potentially result in insomnia symptomatology through sleep disturbance [8•].

There are many determinants of insomnia or insomnia-like conditions. Approximately 35 to $49 \%$ of the US adult population have complaints of problems falling asleep or have daytime sleepiness; however, this is not necessarily the same as a clinical diagnosis of insomnia, which can affect approximately 4-22\% depending upon the diagnostic nosology [9].

Insomnia is an under-recognized condition for several reasons. Epidemiological research suggests that the prevalence of insomnia symptoms is $15-30 \%[10 \bullet, 11 \bullet]$. Yet despite this high prevalence, only $15-40 \%$ with insomnia have discussed their insomnia symptoms with their healthcare provider [12•, 13-15]. While cost and access to care clearly serve as barriers for many individuals with insomnia symptoms, as with many other medical disorders, other additional factors may play a role.

First, low rates of medical awareness and medical literacy related to insomnia consequences and treatment options may result in an under-reporting of insomnia symptoms by patients. Second, mental disorders are often associated with blame or stigma [16]. In part for this reason, recognition rates for mental disorders in primary care were only approximately $33 \%$ in one 5-year study [17]. Third, patient-physician communication difficulties have been identified as one risk factor for low recognition rates by healthcare providers. Marvel and colleagues explored these factors in a detailed study of 264 patient-physician interviews in which they recorded time durations for various components of the encounters, including the patient's statement of the chief complaint (opening statement) and physician redirection [18]. Their results were striking: only $28 \%$ of the patients were able to fully complete their presenting complaint, with physicians redirecting the patient after a mere $23.1 \mathrm{~s}$, on average. Late-arising complaints occurred in up to $35 \%$ of the encounters when physicians redirected the patients' initial complaint. Since insomnia may often occur co-morbid with other conditions, it is likely that insomnia may often be brought up later in the interview, and, thus, be at higher risk of being neglected due to time constraints. A fourth factor that is also physician-related is limited training in sleep disorders, which results in unfamiliarity with insomnia symptom assessment and prescribing sedative-hypnotics [19]. Adams et al. concluded that few healthcare providers have received in-depth training in sleep-related inquiries and it is a contributing factor to larger health issues in their patients [20•].

Shorr et al. reviewed 536 patient encounters during which a sedative was prescribed. Despite the fact that sedatives were ordered during those encounters, only $12 \%$ of the primary care physicians documented sleep histories [21]. A survey of 
935 primary care physicians noted that $57 \%$ of the intake questionnaires did not include any sleep-related questions [22]. Another inpatient study noted that while $47 \%$ of patients reported insomnia complaints on a research questionnaire, chart review revealed that none of the formal inpatient records mentioned these symptoms [23•].

There is clearly a need to develop new methods to address this significant gap in patient care. The feasibility of using internet-based web portals for the screening of common health conditions has been explored [24, 25]. One study invited 4047 current users of an existing patient healthcare portal system (Patient Site at Beth Israel Deaconess Medical Center, Boston) [24]. Of this cohort, 2113 opened the invitation and 1001 consented, with 319 subsequently screening positive for depression, chronic pain, or mobility problems. The authors also noted that overall there was a high rate of acceptance for reporting health concerns using the internet.

\section{Participatory Healthcare}

Participatory healthcare seeks to enhance patient involvement in medical evaluations and treatments. It offers several potential avenues of intervention that can attempt to address the under-recognition of insomnia symptoms. A broad range of terms have been used to describe this process, including "health coaching", "patient activation", and "shared-decision making" [26-29].

Prior participatory healthcare research that has focused on specific components, such as "preparing the patient for contact with a care provider," has had mixed results [26, 30, 31]. One study examining the use of a pre-encounter self-completed agenda form (SCAF) found no significant difference in prescription rate or patient satisfaction [31]. Another randomized trial using a patient-completed agenda form noted that the number of problems identified during the encounter increased as did patient satisfaction [32]. Espallargues et al. [30], when reviewing eleven interventions related to mental health, noted that there was a higher rate of diagnosis (combined odds ratio $(\mathrm{OR})=1.91$ (95\% confidence intervals 1.28-2.83).

\section{Methodology}

For the literature review, PubMed, Google Scholar, Psych Info, and Medline databases were used to search for peerreviewed articles using the following terms: mobile devices, smartphones, insomnia prevalence, insomnia correlates, insomnia determinants, insomnia treatment options. and insomnia prevention. The searches were limited to the time frame from 1992 to 2015. After the initial literature search, 46 citations were reviewed for eligibility. Forty-six eligible article abstracts were printed out and carefully reviewed. Twenty- five articles made the final eligibility, and full PDF article files were accessed.

\section{Risk of Insomnia from Mobile Device Use}

Adams and Kisler studied technology use among college students and symptoms of insomnia, depression, and anxiety. It was anticipated that a higher level of technology use after the onset of sleep predicted poorer sleep quality which further predicted symptoms of depression and anxiety [20•]. The most revealing research finding here indicated that time awake due to technology use, rather than the volume of phone calls or texts, predicted sleep impairment. The most vulnerable are those college students who find it difficult to set boundaries around technology use and are at increased risk for mental health concerns in the future [20•].

A study by Arora et al. tested the association of weekday bedtime use of six technologies with various sleeping parameters: sleep quality, sleep duration, frequent nightmares, sleepwalking frequency, and cognitive difficulty. The results indicated music listeners were at increased risk for frequent nightmares whereas mobile phone use was significantly associated with difficulty falling asleep [33]. A study by Fossum et al. also found a positive correlation between mobile device use in bed for any recreational use and severity of insomnia symptoms particularly after lights out [8•]. A study by Lemola et al. revealed that adolescents' electronic media use at night is associated with sleep disturbance as a "partial" mediator towards the outcome of depressive symptoms [34]. The National Sleep Foundation's 2011 Sleep in America Poll in a study by Gradisar et al. revealed that the number of technological devices used in the bedroom before bedtime within the hour before bed was significantly related to difficulty falling asleep [35]. This was after controlling for covariates for passive distractions (TV, reading, and $\mathrm{mp} 3$ players) [35]. On the contrary, Brunborg et al. found no such association between mobile phone use in the bedroom and symptoms of insomnia [8•].

A study by Dunker-Hopfe et al. examined the effects of electromagnetic fields and non-electromagnetic fields' effects of mobile phone-based stations. They performed an experimental, double-blind, sham-controlled field study measuring sleep quality in Germany, and the results indicated significant negative impact on sleep quality when exposed to mobile phone-based stations alone but not necessarily electromagnetic fields themselves [36]. Another study by Fritzer et al. examined effects of short- and long-term pulsed radiofrequency electromagnetic fields on night sleep and cognitive functions in healthy young male subjects. They found no significant 
effects on night sleep or cognitive functions investigated by an array of neuropsychological tests [37].

\section{Assessment of Sleep Disorders}

While mobile devices have the potential to disrupt sleep, they also offer unique opportunities for assessment and management. Consumer-oriented wrist-worn actigraphy and accelerometry units are now being used in clinical settings to measure basic sleep patterns, such as number of wakings, hours slept, and sleep efficiency [38]. The use of smartphone apps in reporting over long durations is also found to be feasible as a recent study revealed sustained high compliance rates of daily self-reporting sleep-disturbance data over a 90day period in breast cancer patients [38]. In addition to diagnostic and therapeutic potential, apps are also being used to deter insomnia and promote good sleep behaviors [38]. Shirazi et al. noted sharing sleep information after capturing sleep duration via social networks increases healthier sleep habits [39•].

The gold standard for assessment of sleep is polysomnography which combines all-night electroencephalogram (EEG) with eye movements and muscle tones but requires specialized equipment, trained technicians, and a dedicated sleep laboratory [38]. The mobile phone's sleep-related apps use a much more basic approach that primarily measures presence or absence of the limb movement through internal accelerometer recordings to infer wakefulness and sleep patterns [40].

Insomnia can be a symptom of sleep-disordered breathing. Garde et al. have also examined the potential use of the smartphone oximeter with pulse rate variability as an athome screening tool to monitor for pediatric sleep disordered breathing over multiple nights, and they noted promising test characteristics (sensitivity of $88.4 \%$, specificity of $83.6 \%$, positive predictive value of $76.0 \%$ ) [41].

Nakano et al. noted that smartphone-based snoring sound monitors can be used to effectively monitor snoring in obstructive sleep apnea (OSA) patients in a controlled laboratory setting. The diagnostic sensitivity and specificity of the smartphone for diagnosing OSA was 0.70 and 0.94 , respectively, and snoring measured by the smartphone for diagnoses correlated with snoring time measured by polysomnography $(r=0.93)$ [42]. Another study by Shin et al. in an at-home setting revealed snoring detection through a smartphone app could have a high sensitivity and specificity (98.6 and 94.6\%, respectively); however, the positive predictive value was only $70.4 \%$ most likely due to the naturalistic setting with background noises, such as car traffic [43].

Other mobile devices such as wearable photoplethysmographic (PPG) sensors have emerged as a novel therapeutic option in preventing sudden cardiac deaths due to syncope in recent randomized clinical trials [44].

\section{Use of Mobile Device for the Treatment of Insomnia}

A search of the available apps reveals several hundred that are related to insomnia treatment. However, few have been independently verified to confirm efficacy, and no published peerreviewed randomized controlled trials were found. We will briefly review the broad categories of apps aiming to improve sleep quality or duration: apps for relaxation and falling asleep, apps to track and record a sleep diary or daily sleep $\log$, and apps for specific sleep problems [45].

There are apps available to assist people in falling asleep. These apps may utilize realization techniques and provide the user with soothing sounds, such as those found in nature or calm music [45]. Apps may even provide guided meditation, breathing exercises, or hypnosis in an effort to help people fall asleep [45]. Finally, apps have also been developed that implement cognitive-behavioral therapy for insomnia or can be used as adjuncts to web-based cognitive-behavioral therapy programs.

Once someone is asleep, there are mobile applications for sleep tracking and recording sleep diaries or sleep logs. These apps allow people to record and monitor their sleep, and they may use the accelerometer in the phone or separate hardware to track movement, wakefulness, and sleep patterns throughout the night [45]. Some of these apps are purely for monitoring and writing about one's sleep while others attempt to determine the best time for the user to wake up or provide an analysis of the persons sleep [45].

In addition to the two large categories of apps discussed, there are miscellaneous other apps that target very specific problems that those with insomnia might face. There are applications for monitoring snoring, which allow the user to record episodes of snoring and to learn how frequently they are snoring and to play back the recordings later [46]. There are also applications available that focus on the blue light emitted from mobile phones. An application can be used to reduce the harshness of the light coming from the phone when used at night [45]. There are many other miscellaneous applications that target various aspects of sleep, and there are more sleep-related applications being developed everyday [45].

\section{Conclusion}

There is a clear need to conduct additional research examining the role of mobile devices for the assessment and treatment of insomnia. There are many sleep-related mobile applications online, but most are not tested, and yet some have been downloaded over a million times. This is of particular 
relevance because of the ever increasing penetration of mobile devices across all ages, sexes, ethnicities, geographic locations, and socioeconomic statuses not just in the USA but throughout the world. The novel approaches of these apps, if backed by scientifically researched and validated concepts, have the potential to improve assessment and treatment of insomnia by patients and providers. This will eventually expand the horizon of insomnia-related therapeutic approaches while simultaneously lessening the burden of its under-recognition, particularly through the potential for increased participatory healthcare.

Acknowledgments Support for Rebecca Nock: National Institute of Nursing Research, Ruth L. Kirschstein: National Research Service Award (NRSA) in Individualized Care for At Risk Older Adults (T32NR009356), PI: Drs. Naylor and Bowles

\section{Compliance with Ethical Standards}

Conflict of Interest Mohammed N. Khan, Rebecca Nock, and Nalaka $\mathrm{S}$. Gooneratne declare that they have no conflict of interest.

Human and Animal Rights and Informed Consent This article does not contain any studies with human or animal subjects performed by any of the authors.

\section{References}

Papers of particular interest, published recently, have been highlighted as:

- Of importance

1. Smith A. Smartphone ownership - 2013 update. [internet] 2013. Available at: http://www.internetretailer.com/commentary/2013/04/ 01/smartphones-are-spearheading-commerce-revoltion.

2. Siwiki B. Smartphones are spearheading a commerce revolution 2013. Available at: http://www.internetretailer.com/ commentary/2013/04/01/smartphones-are-spearheadingcommerce-revolution.

3. Kwon N, Colucci A, Gulati R. A survey of the prevalence of cell phones capable of receiving health information among patients presenting to an urban emergency department. J Emerg Med. 2013;44(4):875-88. Approximately 90\% of the cell phone owners having the device with them during their emergency room visit.

4. Americans' Views on Privacy and Security. 2014. Retrieved June 24, 2015, from http://www.pewinternet.org/.

5. Middlemass J, Davy Z, Cavanagh K, et al. Integrating online communities and social networks with computerised treatment for insomnia: a qualitative study. Br J Gen Pract. 2012;62(602):840-50. Mobile device use well suited for Qualitative research in insomnia assessment.

6. Diagnostic and Statistical Manual of Mental Disorders. 5th Ed. DSM-5 Paperback - May 27, 2013. 2013. Retrieved June 24, 2015.

7. Home | Psychiatry.Org. 2000. Retrieved June 24, 2015, from http:// psychiatry.org/.
8. Fossum I, Nordnes L, et al. The association between use of electronic media in bed before going to sleep and insomnia symptoms, daytime sleepiness, morningness, and chronotype. Behav Sleep Med. 2014;12(5):343-57. doi:10.1080/15402002.2013.819468. The key definition and three main factors describing insomnia are discussed here.

9. Roth T, Coulouvrat C, Hajak G, Lakoma M, Sampson N, Shahly V, Kessler R. Prevalence and perceived health associated with insomnia based on DSM-IV-TR; international statistical classification of diseases and related health problems, Tenth revision; and research diagnostic criteria/international classification of sleep disorders. Biol Psychiatr. 2011; 592-600.

10. Grandner M, Martin J, Patel N, et al. Age and sleep disturbances among American men and women: data from the US behavioral risk factor surveillance system. Sleep. 2012;35(3):395-406. Several reasons of insomnia's under-recognitions are mentioned here.

11. Ohayan M. Epidemiology of insomnia: what we know and what we still need to learn. Sleep Med Rev. 2002;6(2):97-111. Epidemiological reasons for insomnia under-recognitions are mentioned here.

12. Gooneratne N, Tavaria A, Patel N. Perceived effectiveness of diverse sleep treatments in older adults. J Am Geriatr Soc. 2011;59(2): 297-303. Only 15-40\%with insomnia has discussed their insomnia symptoms with their health care provider.

13. Leger D, Poursain B. An international survey of insomnia: underrecognition and under-treatment of a polysymptomatic condition. Curr Med Res Opin. 2005;21(11):1785-92.

14. Bailes S, Baltzan M, Rizzo D. Sleep disorder symptoms are common and unspoken in Canadian general practice. Fam Pract. 2009;26(4):294-300.

15. Bartlett D, Marshall N, Williams A, Grunstein R. Predictors of primary medical care consultation for sleep disorders. Sleep Med. 2007;9 857-64.

16. Lawrence V, Banerjee S, Bhugra D. Coping with depression in later life: a qualitative study of help-seeking in three ethnic groups. Psychol Med. 2006;36(10):1375-83.

17. Jackson J, Passamonti M, Kroenke K. Outcome and impact of mental disorders in primary care at 5 years. Psychosom Med. 2007;69(3):270-6.

18. Marvel M, Epstein R, Flowers K, et al. Soliciting the patient's agenda: have we improved? J Am Med Assoc. 1999;281(3):283-7.

19. Israel A, Lieberman J. Tackling insomnia: diagnostic and treatment issues in primary care. Postgrad Med. 2004;116(6):7-13.

20. Adams K, Kisler T. Sleep quality as a mediator between technology-related sleep quality, depression, and anxiety. Cyberpsychol Behav Soc Netw. 2013;16(1):25-30. Physician's lack of in-depth training in recognizing sleep disorders contributing to larger health issue.

21. Shorr R, Bauwens S. Diagnosis and treatment of outpatient insomnia by psychiatric and nonpsychiatric physicians. Am J Med. 1992;93(1):213-23.

22. Sorscher A. How is your sleep: a neglected topic for health care screening. J Am Board Fam Med. 2008;21(2):141-8.

23. Meissner H, Reimer A, Santiago S. Failure of physician documentation of sleep complaints in hospitalized patients. West J Med. 1998;169(3):146-9. Physicians do not document sleep related problems are often in the charts.

24. Leveille S, Huang A, Tsai S. Screening for chronic conditions using a patient internet portal: recruitment for an internet-based primary care intervention. $\mathrm{J}$ Gen Intern Med. 2008;23(4):472-5.

25. Lin C, Bai Y, Lui C. Web-based tools can be used reliably to detect patients with major depressive disorder and subsyndromal depressive symptoms. BMC Psychiatr. 2007;7:12. 
26. Leveille S, Huang A, Tsai S. Health coaching via an internet portal for primary care patients with chronic conditions: a randomized controlled trail. Med Care. 2009;47(1):41-7.

27. Wensing M, Baker R. Patient involvement in general practice care: a pragmatic framework. Eur J Gen Pract. 2003;9(2):62-5.

28. Wensing M, Grol R. Patients' views on healthcare. A driving force for improvement in disease management and health outcome. Dis Manag Health Outcome. 2000;7(3):117-25.

29. Wetzels R, Harmsen M, Wensing M, Weel C, Grol R. Interventions for improving an older patient's involvement in primary care episodes. Cochrane Database Syst Rev Protoc. 2007; (1).

30. Espallargues M, Valderas J, Alanso J. Provision of feedback on perceived health status to health care professionals: a systematic review of its impact. Med Care. 2000;38(2):174-86.

31. Hamilton W, Russell D, Stabb C, Seamark D, Campion-Smith C, Britten N. The effect of patient self-completion agenda forms on prescribing and adherence in general practice: a randomized controlled trial. Fam Pract. 2007;24(1):77-83.

32. Middleton J, McKinley R, Gillies C. Effect of patient completed agenda forms and doctors' education about the agenda on the outcome of consultations: randomised controlled trial. BMJ. 2006;332(7552):1238-42.

33. Arora T, Broglia E, et al. Associations between specific technologies and adolescent sleep quantity, sleep quality, and parasomnias. Sleep Med. 2014;15(2):240-7.

34. Lemola S, Perkinson-Gloor N, et al. Adolescents' electronic media use at night, sleep disturbance, and depressive symptoms in the smartphone age. J Youth Adolesc. 2015;44(2):405-18.

35. Gradisar M, Wolfson A, Harvey A, Hale L, Rosenberg R, Czeisler C. The sleep and technology use of Americans: findings from the National Sleep Foundation's 2011 sleep in America poll. J Clin Sleep Med. 2013;9(12):1291-9.

36. Danker-Hopfe H, Dorn H, et al. Do mobile phone base stations affect sleep of residents? Results from an experimental double- blind sham-controlled field study. Am J Hum Biol. 2010;22(5): 613-8.

37. Fritzer G, Göder R, Friege L, Wachter J, Hansen V, Hinze-Selch D, et al. Effects of short- and long-term pulsed radiofrequency electromagnetic fields on night sleep and cognitive functions in healthy subjects. Bioelectromagnetics. 2007;28(4):316-25.

38. Min YH, Lee JW, et al. Daily collection of self-reporting sleep disturbance data via a smartphone app in breast cancer patients receiving chemotherapy: a feasibility study. J Med Internet Res. 2014;16(5):e135.

39. Shirazi AS, Clawson J, Hassanpour Y, Tourian MJ, Schmidt A, Chi EH, et al. Already up? Using mobile phones to track \& share sleep behavior. Int J Hum Comput Stud. 2013;71:878-88. Sharing sleep information after capturing sleep duration via social networks increases healthier sleep habits.

40. Behar J, Roebuck A, et al. A review of current sleep screening applications for smartphones. Physiol Meas. 2013;34(7):29-46.

41. Garde A, Dehkordi P, et al. Development of a screening tool for sleep disordered breathing in children using the phone Oximeter. PLoS ONE. 2014;9(11):e112959.

42. Nakano H, Hirayama K, et al. Monitoring sound to quantify snoring and sleep apnea severity using a smartphone: proof of concept. J Clin Sleep Med. 2014;10(1):73-8.

43. Shin $\mathrm{H}$, Cho J. Unconstrained snoring detection using a smartphone during ordinary sleep. Biomed Eng Online. 2014;13:116.

44. Meyer C, Carvalho P, Brinkmeyer C, Kelm M, Couceiro R, Mühlsteff J. Wearable sensors in syncope management. Med Sci Monit Int Med J Exp Clin Res. 2015;21:276-82. doi:10.12659/ MSM.892147.

45. Van den Bulck J. Sleep apps and the quantified self: blessing or curse? J Sleep Res. 2015;24:121-3.

46. Camacho M, Robertson M, Abdullatif J, Certal V, Kram YA, Ruoff CM, Brietzke SE, Capasso R. Smartphone apps for snoring. J Laryngol Otol. 2015; 1-6. 